Revue internationale de pédagogie de

l'enseignement supérieur

28(1) | 2012

Numéro spécial - printemps 2012

\title{
Les métiers de l'éducation et de la formation : une professionnalisation en tensions
}

Philippe Maubant et Lucie Roger

\section{(2) OpenEdition \\ Journals}

Édition électronique

URL : http://journals.openedition.org/ripes/593

ISSN : 2076-8427

Éditeur

Association internationale de pédagogie universitaire

Référence électronique

Philippe Maubant et Lucie Roger, « Les métiers de l'éducation et de la formation : une

professionnalisation en tensions », Revue internationale de pédagogie de l'enseignement supérieur [En

ligne], 28(1) | 2012, mis en ligne le 20 avril 2012, consulté le 07 septembre 2020. URL : http://

journals.openedition.org/ripes/593

Ce document a été généré automatiquement le 7 septembre 2020

Article L.111-1 du Code de la propriété intellectuelle. 


\title{
Les métiers de l'éducation et de la formation : une professionnalisation en tensions
}

\author{
Philippe Maubant et Lucie Roger
}

\section{Introduction}

1 La question de la professionnalisation est aujourd'hui une problématique centrale constitutive des réformes des curricula de formation professionnelle dans les métiers adressés à autrui. En nous appuyant sur les travaux de Piot (2009), nous définissons les métiers adressés à autrui comme des activités qui requièrent l'adhésion du sujet, objet de l'intervention et qui visent sa transformation, comme les métiers d'interactions humaines: enseigner, former, soigner, accompagner, aider, conseiller, assister, animer... Pour définir ces métiers adressés à autrui, Piot (2009) rappelle que trois éléments sont importants à prendre en compte sont: 1) le collectif de travail ; 2) l'environnement institutionnel ; 3) le contexte organisationnel. Il souligne que deux registres de compétences sont en tension: d'une part, l'objet de service (contenus d'enseignement ou de formation, procédures et gestes de soin, connaissances juridiques en travail social par exemple) et d'autre part des compétences communicationnelles qui, comme le stipulent Couturier et Chouinard (2008), sont le creuset des compétences de l'activité.

2 L'analyse des travaux récents sur la professionnalisation révèle deux visées différentes : l'une questionne l'évolution des métiers tant du point de vue de l'identité professionnelle que des pratiques mises en œuvre ou des compétences à mobiliser ; la seconde s'intéresse à l'évolution des formations professionnelles préparatoires à ces métiers. Pour comprendre les différents sens des discours sur et pour la professionnalisation dans les métiers adressés à autrui, nous avons choisi de centrer notre réflexion sur les métiers de l'éducation et de la formation. Dans cette perspective, nous avons souhaité recourir à différentes grilles de lecture permettant de révéler les 
valeurs ajoutées attendues de la professionnalisation dans ces métiers de l'éducation et de la formation, qu'elles soient socio-professionnelles, pédagogiques, organisationnelles.

3 Mais pour réaliser cette entreprise de compréhension, nous devons examiner tout d'abord en quoi cette problématique révèle un long processus de recherche de reconnaissance de professions tant d'un point de vue de la constitution d'un groupe professionnel que d'un point de vue de la mise à jour et de l'analyse des tâches professionnelles caractéristiques de ces professions. Dans une seconde partie, ce texte cherchera à définir les termes suivants: professionnalisation, professionnalité et savoirs professionnels. Dans une dernière partie, nous tenterons d'identifier les conditions de mise en œuvre et de réussite d'un processus de professionnalisation. Nous verrons également, de quelles manières les universités québécoises cherchent à s'inscrire dans une visée de professionnalisation de l'éducation et de la formation.

\section{2. Évolution historique des discours sur et pour la professionnalisation dans les métiers de l'éducation et de la formation}

4 À l'instar de Carbonneau (1993), nous pouvons considérer que cette valorisation progressive des professions prend racine dans l'espoir suscité au $19^{\text {ème }}$ siècle par le développement de professions cherchant à régler «les problèmes humains de tous ordres » (p. 84). Jusque dans les années 60, aux États-Unis, nous assistons à une apologie des professions et de facto à l'essor d'une idéologie, voire d'une culture des professions avec comme corollaire une foi incontestée dans les savoirs scientifiques comme remèdes universels aux problèmes sociaux.

5 L'étude des professions s'appuie alors sur une analyse des tâches et sur une étude approfondie des processus en jeu dans l'activité professionnelle avec comme principale visée la recherche systématique d'une rationalité et d'une efficacité de l'action. Ainsi, cette nouvelle culture des professions (Champy, 2009) cherche à répondre à un besoin de croissance de la richesse issue du travail. Ce capitalisme du travail en plein essor considère qu'en analysant l'activité professionnelle, il sera, dès lors, possible d'améliorer la pratique et a fortiori les performances, la productivité et la rentabilité des travailleurs. La société nord-américaine encourage donc le développement des professions, car celles-ci soutiennent son essor économique. Néanmoins, à partir des années 1960, quelques incidents vont mettre en évidence les excès de certaines pratiques professionnelles. Ils marquent un début de rupture de confiance entre les professions, d'une part, et la population, d'autre part, révélant des formes d'aveuglements liés à une foi sans faille, et sans doute excessive, dans le progrès technique et les limites d'une apologie sans nuance des savoirs scientifiques. Ces évènements mettront aussi en exergue combien certaines professions vont chercher à faire passer leurs propres intérêts avant ceux de la population. Dès cette époque, de vives attaques contre certaines professions, la médecine, le droit, vont voir le jour et des critiques de plus en plus vives vont apparaitre prenant principalement comme cible les discours sur et pour la professionnalisation.

6 La principale de ces critiques est incarnée par Illich (1977). Il dénonce notamment la perte de pouvoir de décision et d'action des citoyens face à des professions 
dominatrices. Carbonneau (1993) reprend cette idée lorsqu'il affirme combien certaines de ces professions ont fait alliance avec l'État qui « à travers divers systèmes de prise en charge de la population (éducation, protection sanitaire, sociale, légale) oblige chacun à passer par les professions concernées » (p. 87). Nous pouvons comprendre aisément que cette lecture critique des professions soit présentée comme un contre-point des discours zélateurs et enthousiastes sur les professions du $19^{\text {ème }}$ siècle. Mais cette critique devient plus précise lorsqu'elle s'intéresse aux pratiques professionnelles et aux savoirs qui y sont associés. La question des savoirs de ces professions, l'analyse de leurs fondements épistémologiques, du sens et de la nature du rapport au savoir et aux savoirs de ces professions servent de prétextes à contester leur survalorisation dans la société. En effet, les savoirs de ces professions fondées pour la plupart sur la science constituent des objets de critiques et de polémiques. Des experts mettent en cause notamment la domination que ces professions tirent de ces savoirs et de leurs maittrises et s'en prennent aux rapports de pouvoir qu'ils établissent ainsi avec la population. Ces auteurs ne remettent pas en cause ce qui constitue la raison d'être opératoire d'une profession, mais bien l'apologie excessive de celle-ci par la société. Comme le note Carbonneau (1993), «cette reconnaissance d'une profession est due non au hasard d'une faveur sociale, mais à un processus politique de contrôle du marché et des conditions de travail, acquis par un groupe social à un moment historique déterminé " (p. 90). Au-delà des enjeux de pouvoirs que peuvent contenir certains discours sur et pour les professions, nous pouvons noter, notamment dans certains secteurs professionnels, comme l'agriculture par exemple (Boulet, 2001), que cette démarche laudatrice des professions trouve son sens dans une tradition culturelle où certains métiers constituant les fonds baptismaux d'une société traditionnelle se doivent d'être magnifiés. À l'instar de Barreau (2001), il est intéressant de rappeler ici combien des gouvernements (prenons l'exemple du gouvernement français de Vichy en 1940) ont su tirer profit de cet encensement de certains de ces métiers pour asseoir leur légitimité dans la vénération d'un certain passé et le recours dithyrambique aux valeurs traditionnelles. Mais pour que ces professions puissent s'installer de manière pérenne dans des formes de pouvoir, encore faut-il les légitimer non seulement par la nature des savoirs professionnels qui les fondent, mais aussi par les lieux-ressources desquels ces savoirs sont extraits.

7 C'est ainsi que la fin du $19^{\text {ème }}$ siècle marque la valorisation du principe de la méritocratie et la prise en considération de sa figure emblématique: le diplôme. Chaque groupe professionnel souhaitant gagner, voire préserver des formes de légitimité et de pouvoir visera, dès lors, à asseoir la professionnalisation du métier en la fondant sur une formation professionnelle dans laquelle il cherchera à maintenir certaines formes de contrôle. Dans l'Angleterre du début du $20^{\text {ème }}$ siècle et dans les pays anglo-saxons en général, le retrait de l'État du contrôle de l'accès à certaines de ces professions (Bourdoncle, 1991) des écoles professionnelles appartenant à des groupes professionnels se développera. En France, la naissance d'une noblesse d'État (Bourdieu, 1989), depuis le $19^{\text {ème }}$ siècle, conduit à l'établissement de groupes professionnels relevant de l'État et soumis bien sûr aux décisions politiques des différents gouvernements. L'essor d'ordres professionnels est très symptomatique de cette tendance. Même si ces professions, protégées par ces ordres professionnels, visent à s'assurer d'instances de représentativité dans les nouvelles organisations sociales, elles cherchent aussi, par le recours à des formations professionnelles, à approfondir leur expertise technique, voire scientifique. C'est donc tout d'abord le statut et la fonction 
sociale des professions dans la société qui semblent constituer les enjeux des discours sur et pour les professions. Ces deux dimensions sont caractéristiques des rapports de pouvoir qui se constituent entre les groupes sociaux, en particulier entre les différents groupes professionnels. Certes, l'histoire de la création de ces groupes professionnels et de leur essor joue incontestablement un rôle dans la place qu'ils occupent aujourd'hui. Mais ces places sont en constante évolution au regard aussi de la transformation des attentes sociales. Ce sont d'ailleurs ces attentes sociales qui interpellent les professions dans leurs capacités à résoudre les problèmes contemporains, qu'il s'agisse de problèmes éducatifs, de questions de santé ou de risques touchant à la sécurité intérieure d'une nation. Pour soutenir ces questionnements et y trouver des réponses, on se tourne alors vers les professions en interrogeant les savoirs professionnels qu'elles mobilisent. Il s'agit, ici, de vérifier la pertinence des savoirs professionnels maitrisés et leur adéquation efficace et rationnelle aux réalités des situations de travail. En interrogeant cette pertinence des savoirs professionnels au regard d'une visée d'expertise, d'efficacité, de rationalité et d'efficience, on cherche aussi à interroger la place sociale et politique qu'occupent ces professions. Dans cette perspective, on parle aujourd'hui de savoirs professionnels (Vanhulle, 2008), de compétences (Mayen, 2008), de savoir d'experts (Carbonneau, 1993). Les théories de l'action (Baudouin \& Friedrich, 2001), celles sur la réflexion en action (Schön, 1991), les importants apports de la psychologie du travail (Clot, 2006) et de l'ergonomie (Leplat, 1992), puis plus récemment de la didactique professionnelle (Pastré, 1997), les différentes typologies sur les savoirs (Barbier, 1996) contribuent à identifier et à analyser les savoirs en jeu dans les actes professionnels. L'intention de lire et de comprendre les savoirs professionnels se trouve aussi enrichie d'une visée plus ambitieuse, celle de parvenir à construire une épistémologie de la pratique professionnelle. Cherchant à échapper à une stricte lecture analytique des actes professionnels, fondée sur une conception d'une rationalité technique empruntant au bureau des méthodes (Fabre, 1994) et dont l'ingénierie de formation se revendique souvent, la recherche d'une épistémologie, voire d'une théorie de la pratique professionnelle constitue aujourd'hui une manière d'interroger les discours sur les professions en ramenant ceux-ci vers une compréhension de leurs usages sociaux. En posant la question de ce qui fonde une pratique professionnelle, on interroge de fait les processus de construction et de développement de la pratique. On mobilise aussi une réflexion sur les dispositifs professionnalisants et sur les situations formatives préparant à l'exercice de cette pratique.

8 Nous voyons donc qu'ici l'analyse des discours sur et pour les professions conduit à identifier quelques zones d'ombre et enjeux tant pour les professionnels eux-mêmes que pour la société dont l'évolution économique et politique accompagne dans un dialogue permanent les transformations des groupes professionnels et des pratiques qui les caractérisent. Cette analyse permet aussi de révéler les deux pôles de la sociologie des professions qui, tour à tour, s'intéressent aux théories générales sur les professions puis aux approches plus singulières permettant de caractériser les constituants d'une profession au regard des spécificités des actes professionnels. D'une période et d'un contexte favorable à l'idéalisation des professions, en particulier le 19 ème siècle dans les pays anglo-saxons, à une quasi-centration aujourd'hui sur l'analyse de la pratique professionnelle, s'intéresser aujourd'hui à la professionnalisation, c'est chercher fondamentalement à concilier une analyse des discours sur et pour les professions avec une compréhension des pratiques professionnelles constitutives de 
celles-ci. C'est donc moins comme le suggère Carbonneau (1993), viser une prise en compte dialogique entre les théories sur les professions, pensées dans une perspective historique et sociologique, d'une part, et une analyse plus ethnographique de celles-ci, d'autre part, que de chercher à mettre en évidence de quelles manières une analyse des processus de professionnalisation permet d'identifier différentes questions et enjeux touchant aux statuts des professions et aux dispositifs et processus de formation professionnelle préparatoires à l'exercice professionnel.

\section{Des termes à définir dans les métiers de l'éducation et de la formation : professionnalisation, professionnalité, et savoirs professionnels}

9 Le terme de professionnalité fait son apparition dans différents textes, y compris dans des textes d'institutions ministérielles traitant du champ par exemple de la formation des enseignants comme le rapport Bancel (1989) en France ou dans le référentiel de formation à l'enseignement du gouvernement du Québec en 2001. Le rapport Bancel définissant ce qui allait constituer en France la création des Instituts universitaires de formation des maîtres (IUFM) «a tenté de déterminer la professionnalité requise pour enseigner en indiquant les axes du processus de professionnalisation à mettre en œuvre afin d'atteindre cet objectif» (PRISME, 2008). Ce rapport incarne un choix de conception et de structuration d'un programme de formation fondées sur une nouvelle conception de l'activité professionnelle des maîtres : l'approche par compétences. Cette nouvelle perspective, présente aujourd'hui dans les programmes de formation des maitres tant en France, qu'en Suisse ou au Québec, cherche à favoriser le passage entre une logique de qualification technique vers une logique de compétences. Nous retrouvons aussi cette perspective dans la formation des formateurs d'adultes en France par exemple (Solar \& Hébrard, 2009). Il y a donc, ici, un lien très étroit à établir entre le processus (la professionnalisation) et le résultat attendu (la professionnalité) dans le sens d'une nouvelle identité voire d'une posture professionnelle à construire. La professionnalité est donc la finalité intrinsèque et essentielle du processus de professionnalisation. À l'instar de Champy (2009), nous pouvons considérer que cette réalité se décline pour d'autres professions. Elle est, de plus, toujours illustrée. d'une part. par les compétences identifiées dans les curriculums de formation des maîtres et. d'autre part. en fonction des savoirs professionnels mobilisés dans l'usage et l'exercice des compétences.

\subsection{Définition de la professionnalité}

Selon Lang (1999), c'est l'activité pédagogique qui semble constituer l'emblème de la professionnalité des éducateurs (formateurs ou enseignants). Puisque cette activité est l'expression de la pratique enseignante, il est, pour Lang, légitime que les curriculums de formation à l'enseignement puissent exprimer cette professionnalité attendue par le biais d'énoncés de compétences, notamment didactiques et pédagogiques. Mais si l'on examine de près cette définition de la professionnalité proposée par Lang, celle-ci «renvoie à l'idée de développement professionnel, c'est-à-dire aux processus de construction et d'approfondissement de compétences et de savoirs nécessaires à la pratique d'un métier» $(1999$, p. 26). En proposant qu'il y ait, dans la construction de la 
professionnalité, à la fois des compétences mais aussi des savoirs nécessaires à l'exercice de la pratique, Lang propose une visée heuristique de recherche d'articulation entre la théorie et la pratique. Il y a donc ici une interprétation de l'activité éducative et/ou formative faisant appel à des compétences mais aussi à des savoirs théoriques et pratiques. La définition de la professionnalité proposée par Lang cherche aussi à associer le processus de construction et de développement de compétences au processus de développement professionnel.

11 Nous pouvons identifier cette perspective en analysant, par exemple, le référentiel de la formation des enseignants mis en œuvre au Québec en 2001, dont les douze compétences identifiées reposent sur approche culturelle de l'enseignement. Cette approche singulière vise à former des maîtres qui seront à la fois héritiers, critiques et interprètes (gouvernement du Québec, 2001). En faisant référence au concept de culture, le ministère de l'Éducation propose de faire référence à tous les contenus des programmes d'enseignement du primaire et du secondaire visant la construction d'un rapport entre l'élève et le monde, c'est-à-dire la mise en place d'un «ensemble de situations et de relations dans lesquelles est engagé l'élève pour entrer en relation avec la culture » (Charlot, 1997). On notera, ici, que rien n'est mentionné dans le référentiel de formation à l'enseignement pour préparer les futurs maîtres à être des héritiers, critiques et interprètes. En France, les nouvelles compétences définissant la professionnalité attendue chez les futurs enseignants sont définies dans l'arrêté ministériel du 19 décembre 2006. Nous retrouvons cette définition de la professionnalité dans un texte du collectif IUFM d'Aix-Marseille. Ce texte précise que «si la professionnalité implique nécessairement de former les enseignants à l'acquisition de savoirs multiples, de développer les compétences institutionnellement définies, elle doit également, dans l'exigence d'une qualité de formation au métier, permettre d'articuler les savoirs issus de la recherche et les savoirs professionnels " (Collectif IUFM d'Aix-Marseille, 2009, para. 5). Ici, l'atteinte de la professionnalité passe par un dialogue entre différentes formes de savoirs et doit s'appuyer aussi sur les savoirs issus de la recherche en sciences de l'éducation. Dès lors, dans la situation française, la professionnalité n'est donc pas qu'une recherche de développement de compétences et d'acquisition de savoirs, elle dépend de la capacité de la formation professionnelle à créer les conditions d'une articulation, voire d'un dialogue entre les différentes formes de savoirs et d'une collaboration entre les différentes sources de savoirs. Ainsi, le projet de professionnalité, tel que le proposent Vanhulle et Lenoir (2005), renvoie bel et bien l'éducateur en développement à des choix rationnels fondés sur un dialogue entre des savoirs de la pratique et des savoirs savants issus des sciences de l'éducation (Perrenoud, Altet, Lessard \& Paquay, 2008). Encore convient-il que la formation professionnelle des enseignants ou la formation des formateurs d'adultes puisse créer les conditions d'un tel dialogue. Or, tel que le mentionne Lang (1999), « une académisation de la formation ne suffit plus à promouvoir le modèle du professionnel » (p. 168). Si l'on considère, à l'instar de Hasni et Lenoir (2006), qu'une synergie entre les savoirs théoriques et les savoirs pratiques permet le développement d'une pensée critique à l'égard de la profession et la mise en œuvre d'une réelle pratique réflexive chez l'éducateur en formation (qu'il s'agisse d'un enseignant ou d'un formateur d'adultes), force est de constater que les universités peinent à mettre en place cette synergie. Le processus de professionnalisation visant l'établissement d'une professionnalité de l'intervenant éducatif, pensée comme une nouvelle culture 
professionnelle, requiert donc la mise en dialogue des différents savoirs professionnels mobilisés dans les différentes compétences nécessaires à l'exercice de l'activité.

\subsection{Définition des savoirs professionnels}

Définir les savoirs professionnels n'est pas chose aisée. Nous proposons une définition en trois parties distinctes. Nous distinguerons d'abord les savoirs professionnels des savoirs scolaires. Nous proposerons, dans un deuxième temps, d'identifier les savoirs professionnels selon une perspective tridimensionnelle: théorique, pratique et empirique. Enfin, nous chercherons à mettre en évidence les liens entre savoirs professionnels, professionnalisation et professionnalité à partir d'une lecture des dispositifs de formation professionnelle des formateurs, éducateurs ou intervenants éducatifs.

\subsubsection{Savoirs professionnels et savoirs scolaires}

13 Pour distinguer d'abord les savoirs professionnels des savoirs scolaires, il est d'usage de faire appel aux travaux sur la transposition didactique (Joshua, 1996; Raisky, 1993, 1999 ; Rogalski \& Samurçay, 1994). La transposition didactique, concept initié par Verret (1975), puis développé par Chevallard (1980), pose l'idée que tout savoir se doit de se soumettre à une transformation en vue d'être enseigné ou mis à disposition d'apprenants. Cette transformation s'opère à travers cinq étapes : la désyncrétisation, la dépersonnalisation, la programmation, la publicité et le contrôle. En considérant que les savoirs professionnels doivent, eux aussi, se soumettre à une transposition didactique, nous admettons que les savoirs professionnels, même portés par des professionnels, doivent subir une adaptation pour être des savoirs à enseigner au sein d'un dispositif de formation professionnelle et d'un processus de professionnalisation.

Raisky et Loncle (1993) abordent la question de la désyncrétisation des savoirs professionnels par le biais de la problématique de l'écriture des programmes ou des référentiels de formation. Pour ces deux auteurs, lorsque même ces savoirs professionnels sont formulés dans et par les compétences, les référentiels de formation ne peuvent rendre compte de "l'intervention de l'aléatoire et de l'inscription de ces savoirs dans une ou plusieurs temporalités» (p. 352). La question de la dépersonnalisation, c'est-à-dire $\mathrm{du}$ caractère personnel et singulier des savoirs professionnels, se trouve posée par Lesne (1977) en ces termes : «Réunies pour opérer en commun des reconstructions personnelles de leurs modes d'organisation du réel, le formateur reconstruit dans l'action pédagogique ses représentations scientifiques et ses représentations de l'acte de formation, les formés reconstruisent, quant à eux, leur appropriation du réel avec l'aide du premier» (p. 141). Il semble donc essentiel en formation professionnelle de parvenir à dégager le caractère personnel des savoirs professionnels évoqués pour ne pas les réduire à des routines singulières exclusivement mobilisables par le professionnel qui les identifie. Quant à la question de la programmation des savoirs professionnels, elle se trouve également soulevée par Raisky (1999). Pour cet auteur, la complexité des savoirs professionnels rend nécessaire une modélisation de cette complexité davantage qu'une décomposition linéaire. Ce n'est donc pas en segmentant les compétences et les savoirs professionnels qu'on peut rendre compte des principes organisateurs de la pratique (Bru, 2002) ni de la complexité de l'activité professionnelle. Expliciter celle-ci conduit, nous dit Raisky, à 
penser la pratique professionnelle comme un processus constitué d'interrelations entre des savoirs théoriques, pratiques et techniques et non pas comme une juxtaposition de ces savoirs. Ces trois formes de savoirs constituent selon Raisky les savoirs professionnels. La question de la publicité des savoirs professionnels se trouve soulevée dans les travaux de Rogalski et Samurçay (1994) : «La nécessité d'expliciter des savoirs opérationnels, plus ou moins efficaces, conduit les experts avec lesquels nous travaillons à repérer d'éventuels écarts entre des savoirs en acte et la connaissance individuelle ou collective qu'ils explicitent»(p. 54). Il est donc important, si l'on souhaite identifier et comprendre les éléments constitutifs de l'activité, de repérer les savoirs professionnels à partir de l'explicitation de ces mêmes savoirs. Or, les éducateurs, qu'il s'agisse de formateurs, d'enseignants ou de tout autre intervenant représentatif de métiers relationnels semblent rencontrer des difficultés à définir et à expliquer ce qu'ils font et pourquoi ils le font. Enfin, la question du contrôle est importante à poser tant il semble difficile, voire impossible de saisir non seulement les savoirs professionnels mais surtout les processus de mobilisation, de construction et de mise en acte de ces savoirs au sein de la pratique. Si le contrôle vise à s'assurer de la conformité des savoirs professionnels au regard des attentes de l'institution, l'évaluation des compétences semble plus délicate à mettre en œuvre, car il conviendrait de vérifier non seulement la mobilisation des savoirs professionnels validés par l'institution, mais aussi la capacité du futur professionnel à les convoquer et à les mettre en œuvre à bon escient dans la pratique.

Nous venons de montrer combien une entreprise de transposition didactique des savoirs professionnels vers des savoirs professionnalisants, c'est-à-dire susceptibles de constituer des savoirs à apprendre constitue une démarche délicate qui réclame de toute évidence une autre manière de penser la formation professionnelle dans les métiers adressés à autrui. Mais cela nécessite aussi de proposer une grammaire des savoirs professionnels facilitant leur identification et leur lecture.

\subsubsection{Les trois dimensions des savoirs professionnels}

16 Pour expliciter ces trois dimensions des savoirs professionnels, nous nous référerons principalement aux écrits suivants. Raisky (1993) parle de savoirs scientifiques, pratiques et techniques. Lenoir, Laforest et Pellerin (1995) identifient des savoirs théoriques, pratiques et procéduraux. Mialaret (1996), quant à lui, énonce les savoirs théoriques, les savoirs scientifiques et les savoirs d'action. Roger et Maubant (2011) convoquent les concepts aristotéliciens de theoria, de praxis et de poiesis.

\section{La dimension théorique des savoirs professionnels}

Lenoir, Laforest et Pellerin (1995) identifient des savoirs théoriques et font appel à Malglaive (1990) pour les définir. Ces savoirs sont les connaissances, autrement dit « ce qui est ». Mialaret (1996) propose plusieurs types de savoirs théoriques : le théorique institutionnel (les programmes), l'ensemble des réflexions philosophico-historiques sur l'éducation et l'enseignement et les idéologies pédagogiques ou andragogiques. Rejoignant cette dimension théorique des savoirs professionnels, Raisky (1993) définit les savoirs théoriques comme des savoirs scientifiques. Pour définir ce qu'il entend par savoirs scientifiques, il met en opposition les spécificités du technicien de celles du scientifique: «Le scientifique est animé d'un désir de vérité universellement 
reconnaissable. À ce titre, il ne peut s'agir que d'une vérité en question qui devra, pour se construire, surmonter les obstacles, sans cesse renaissants, parce qu'ils sont dans la conscience même du scientifique » (p. 113). Pour Roger et Maubant (2011), la théorie (Theoria) est à la fois processus de construction et de conception, elle est aussi le produit de cette construction et conception. La théorie est donc à la fois processus intellectuel et objet-produit de ce processus. En somme, nous pouvons constater que la dimension théorique des savoirs professionnels ne se limite pas seulement à un objet extérieur à la pensée, mais bien à un savoir faisant référence à « ce qui est ", c'est-àdire un savoir qui s'intéresse tant au produit qu'au processus cognitif qui participe de sa construction et de son élaboration. Force est de constater que cette dimension théorique des savoirs professionnels, telle que nous la définissons, ici, semble relativement absente des formations professionnelles à visée de professionnalisation en particulier dans les métiers adressés à autrui. Lenoir, Laforest et Pellerin (1995) mentionnent que ces formations conduisent davantage à former des techniciens que des professionnels de la formation ou de l'intervention éducative. Trottier et Lessard (2002) mentionnent, pour leur part, que la formation professionnelle dans les métiers de l'intervention éducative, socio-éducative ou formative est dominée par une vision « centrée sur l'individu et sa psychologie, ainsi que sur les disciplines prises comme des en-soi ou des catégories universelles" (p. 67). Ainsi, la problématique du rapport théorie/pratique qui semble dominer dans les débats sur les formations professionnalisantes ne s'appuie pas sur une définition de la théorie au sens ou nous l'avons présentée plus haut, mais davantage sur une conception d'une théorie de l'action faisant appel principalement au comment éduquer ou comment former? (Roger \& Maubant, 2011).

\section{La dimension pratique des savoirs professionnels}

18 La dimension pratique des savoirs professionnels est d'abord présentée par Raisky (1993) comme les savoirs permettant d'agir. C'est la visée d'efficacité du «fait technique » qui se trouve portée par ces savoirs pratiques. Pour Lenoir, Laforest et Pellerin (1995), la pratique assure l'actualisation des savoirs théoriques et procéduraux. Cette actualisation engage le professionnel dans la totalité de ses actes et de ses pratiques, puisqu'il se situe en interaction contextualisée dans un milieu social où il agit à la fois sur le plan cognitivo-conceptuel (du savoir), sur le plan psychomoteur (du savoir-faire) et sur le plan intentionnel (du savoir-être). Quant à Mialaret (1996), il présente la pratique comme une réponse à la réalité de l'extérieur et la divise en trois niveaux : 1) la réponse impulsive (l'intervenant fait n'importe quoi);2) la réponse plus ou moins adaptée, efficace, mais fermée sur elle-même (l'intervenant utilise des « recettes » qu'il conserve d'une année à l'autre);3) la réponse adaptée, réfléchie, qui nécessite une intense activité psychologique (l'intervenant recherche les solutions les plus pertinentes aux situations éducatives). Il est aussi possible de définir la pratique comme une praxis permettant d'allier l'action et l'activité de l'homme agissant. Roger et Maubant (2011) présentent la pratique comme une incarnation d'une action dont le but est de magnifier le processus de construction des idées (le siège de la theoria) et de pouvoir ainsi rendre ces idées perfectibles. La pratique servirait ainsi en quelque sorte de révélateur à la théorie. Au sein de la formation professionnelle des intervenants dans les métiers adressés à autrui, la dimension pratique des savoirs professionnels semble principalement travaillée pendant les périodes de stage. La difficulté réside à 
exploiter ces périodes de stages comme autant de situations formatives dépassant la simple collecte auprès de maitres de stages ou de superviseurs d'outils, de matériels, de règles de conduite précises et opérationnelles, de routines (Trottier et Lessard, 2002) et pouvant favoriser un dialogue entre les trois dimensions des savoirs professionnels.

\section{La dimension empirique des savoirs professionnels}

19 Lenoir, Laforest et Pellerin (1995) parlent principalement de savoirs procéduraux. Il s'agit, ici, des différentes façons de faire, des procédures, des routines, de ce qu'il faut faire et entreprendre pour agir. L'acquisition de ces savoirs empiriques semble se réaliser en dehors de l'action. Nous serions, ici, dans le domaine des techniques, dans l'univers de ce qui fonctionne dans la réalité sociale et professionnelle, quels que soient les situations et les contextes rencontrés. Les savoirs empiriques, de type procédural, se distingueraient des savoirs d'expérience dans la mesure où les savoirs procéduraux seraient transmis comme des procédures à mettre en œuvre dans des situations spécifiques, relevant de guides d'action garantissant l'efficacité des procédures déployées au regard du diagnostic de la situation professionnelle rencontrée. Pour Raisky (1993), la dimension empirique des savoirs professionnels renvoie aux savoirs techniques. Ils représentent la schématisation de la pratique, c'est-à-dire l'ensemble des démarches qui visent à rendre compte rationnellement des pratiques. Enfin, pour Roger \& Maubant (2011), faisant toujours référence à Aristote, la dimension empirique des savoirs professionnels relève de la poièsis et vise la production matérielle de biens. Il y aurait donc, ici, une finalité productive spécifique constitutive de la dimension empirique des savoirs professionnels.

20 Cette dimension empirique semble très présente dans les dispositifs de formation professionnelle visant à former les formateurs, les gestionnaires de formation ou encore les enseignants. Elle conduit à considérer les connaissances de l'expérience sensible du réel comme des savoirs à part entière. Dans cette perspective, la théorie peut sembler inutile aux enseignants en formation. Ils ne semblent retenir, en fin de compte, que les dimensions empiriques des savoirs professionnels mis en évidence dans le processus de professionnalisation et d'insertion professionnelle.

\subsubsection{Sens et fonctions des savoirs professionnels dans la construction de la professionnalité des enseignants}

21 Comment, à partir d'une définition tri-dimensionnelle des savoirs professionnels, penser et mettre en œuvre un projet de professionnalisation au sein des dispositifs de formation professionnelle dans les métiers adressés à autrui ? Pour répondre à cette question, il est important d'analyser les différentes situations formatives rencontrées par l'éducateur en formation. La compréhension et l'interprétation que l'éducateur novice fait des situations éducatives qu'il rencontre tout au long de son processus de développement professionnel dépendent principalement des savoirs professionnels qu'il a développés en formation, en médiation avec les différents porteurs de savoirs que sont les formateurs, les conseillers pédagogiques, les superviseurs, les collègues de travail... Martineau (2007), s'appuyant sur Schutz, cherche à mettre en évidence un cadre d'intelligibilité des processus de construction de la réalité professionnelle chez l'éducateur débutant. Il propose ainsi le modèle de la typicalité. Ce modèle peut jeter les bases d'une théorie de l'intervention éducative ou formative. Pour Martineau, 
l'individu construit des catégories générales, des types d'expériences, de situations ou de comportements qui constituent des schèmes de référence (des sortes d'idéaux types) et qui lui permettent d'interpréter le social et le culturel. Le monde extérieur n'est pas vécu comme un amalgame d'objets séparés, sans lien dans le temps ou l'espace, mais comme un montage, une organisation plus ou moins cohérente, voire même comme un système. Pour former à l'exercice d'agir professionnellement, il semble nécessaire d'aider le formateur, l'éducateur, l'enseignant à identifier le processus conceptuel organisateur de la pratique. Or, si les énoncés de compétences qui sont présentées dans les programmes de formation ou dans les curricula de formation de formateurs cherchent à définir la professionnalité attendue des futurs intervenants en éducation ou formation, il semble que ce soient les situations formatives soutenant le processus de dialogues entre les différentes formes de savoirs professionnels qui participent de la production de cette professionnalité. Ainsi, rejoignant les questions soulevées par Martineau (2007), il est légitime de se demander ce que savent les futurs experts de l'intervention éducative ou formative des situations formatives qu'ils vivent et dans lesquelles ils développent une intelligence de l'action, une phronesis. Il est important de se demander de quelle nature sont les différents savoirs convoqués et mis en œuvre en situation. En outre, on ne peut pas penser que la formation universitaire à visée professionnalisante dans les métiers adressés à autrui puisse permettre un achèvement de la construction de la professionnalité, car elle se développe tout au long de la carrière du formateur ou de l'enseignant. Mais il faut savoir en formation démontrer que les savoirs professionnels ont un caractère heuristique et interprétatif et conduire, dès lors, les futurs enseignants à mobiliser une pratique réflexive sur leurs savoirs à des fins d'usage dans et pour la pratique.

\section{La visée de professionnalisation des universités québécoises : questions et enjeux}

Si l'on s'appuie sur la réforme de 2001 visant à former à l'enseignement, nous pouvons constater que ce texte vient définitivement « inscrire la formation des maîtres dans une perspective de professionnalisation" (Vanhulle \& Lenoir, 2005, p. 26). Pour justifier cette visée de professionnalisation, les textes officiels du gouvernement du Québec ont rappelé différentes réalités: la diversification des populations scolaires, l'accroissement des problèmes sociaux, de même que les tensions issues des changements technologiques et de la mondialisation de l'économie. Ces facteurs sont pris en compte par les décideurs comme pouvant faire pression sur le travail de l'enseignant. Dès lors, les pouvoirs publics admettent la dimension socialement utile de l'acte d'enseigner. Soulignant la double convergence qui semble s'établir au niveau international, dans les pays de l'OCDE (2008), sur la question de la pratique enseignante et sur celle de la formation à l'enseignement, la réforme québécoise de 2001 propose donc un principe et un concept structurant cette nouvelle formation professionnelle des enseignants : celui de la professionnalisation. Selon ce principe, cette réforme défend et soutient le modèle du maitre professionnel qui doit conduire au développement de l'autonomie professionnelle des enseignants. Le curriculum (gouvernement du Québec, 2001) s'articule autour de douze compétences professionnelles qu'il convient de faire acquérir pendant la formation. Si cette réforme défend un principe: la professionnalisation, elle a une finalité: l'autonomie 
professionnelle des enseignants. Elle s'appuie sur une configuration curriculaire structurée selon une approche par compétences. Dès lors, il semble important d'interroger la lecture et l'interprétation que fera chaque université québécoise de ce principe, de la finalité affichée et revendiquée de l'ingénierie de formation retenue et de la mise en œuvre de la configuration curriculaire en douze compétences.

Pour parvenir à la réussite du processus de professionnalisation, les chercheurs comme Lessard et Tardif (2005) étudiant les différentes évolutions scientifiques, stratégiques des universités québécoises insistent sur l'importance d'améliorer les dispositifs de formation en se centrant tout particulièrement sur les différentes situations formatives rencontrées par les professionnels novices et ceci tout au long d'un cheminement d'insertion et de développement professionnel. Rappelons que dans le référentiel de formation à l'enseignement du Québec, il est précisé que « la mise en contexte à partir de situations pratiques liées à l'exercice de la profession semble être la voie la plus appropriée pour favoriser le développement de compétences et assurer ainsi une plus grande cohérence de la formation » (gouvernement du Québec, 2001, p. 218). Les textes officiels soutiennent donc avec force la nécessité de rechercher des mises en contextes, des formes d'organisation de la formation à partir de situations pratiques sensées soutenir et favoriser le développement des compétences. Ce qui semble être important, ici, c'est l'organisation de la formation qui doit prévoir des situations visant à articuler les différentes formes de savoirs dans la perspective de favoriser le développement de compétences. Mais nous pouvons regretter que peu d'indications soient fournies aux universités pour faciliter ce passage, cette transposition ou ce transfert entre les contenus de formation, les savoirs professionnels à construire et à mobiliser et les compétences à mettre en œuvre et à développer.

Considérant les propositions de Vanhulle et Lenoir (2005), qui appellent à la mise en œuvre d'une "dimension véritablement dialogique de la formation professionnelle » (p. 234), nous adoptons la perspective qu'une formation visant le dialogue entre différents espaces-temps, différents savoirs et porteurs de savoirs pourrait permettre la mise en œuvre du processus de professionnalisation. Or, il conviendrait de soutenir les recherches au Québec portant sur les diverses situations formatives vécues par les professionnels en formation et tout au long de leur développement professionnel leur permettant ainsi de créer les conditions favorables d'un apprentissage professionnel. Certes, des travaux visent à mettre en valeur la fonction et les modalités de fonctionnement de certaines situations comme les périodes de stage, par exemple, ou encore le rôle de certains acteurs dans le dispositif de formation (Correa Molina \& Gervais, 2008). Mais aucun n'étudie la perspective dialogique pouvant se penser et s'organiser entre ces différentes situations au service d'une visée d'apprentissage professionnel, cette visée définissant, à elle seule, le fil conducteur du processus de professionnalisation. Pour étudier cette perspective et cette potentialité dialogique entre les différentes situations présentes en formation, encore faudrait-il retracer la logique qui sous-tend le dispositif de formation et sa structure professionnalisante en étudiant les différentes situations rencontrées par le formateur en formation. Nous pourrions donc proposer d'utiliser le concept de situation pour lire et comprendre les différents contextes formatifs présents dans les dispositifs de formation professionnelle visant à préparer aux métiers adressés à autrui. Nous faisons l'hypothèse que c'est en caractérisant les différentes situations rencontrées par le professionnel en formation, que l'on peut à la fois identifier les capacités dialogiques de ces situations, mais aussi 
repérer les conditions pour faire de certaines d'entre elles et/ou du dialogue entre elles, des occasions d'apprentissage professionnel. Il reste que considérer les situations d'apprentissage professionnel comme situations spécifiques et singulières favorisant le processus de professionnalisation, c'est aussi prendre en compte que le modèle de l'enseignant défendu par le Ministère de l'Éducation au Québec, est bien celui du maître professionnel. L'idée de professionnalisation présente dans différents textes n'est pas qu'un principe déterminant le sens et l'organisation des curricula de formation professionnelle. Elle s'incarne aussi dans la promotion d'un modèle d'éducateur, d'intervenant éducatif, socio-éducatif, formateur : l'intervenant professionnel.

\section{Conclusion}

La visée de professionnalisation contenue dans les dispositifs de formation professionnelle dans les métiers adressés à autrui s'accompagne d'un autre objectif, celui de contribuer à la construction de l'identité professionnelle au travers d'un long processus de développement professionnel. Nous faisons l'hypothèse que cette double orientation, pour qu'elle soit atteinte, doit se traduire sur le plan des finalités, sur le plan de l'organisation et sur le plan des savoirs à apprendre par la mise en œuvre de situations d'apprentissage professionnel (Roger \& Maubant, 2011). Or, Martineau (2008) mentionne que les professionnels de l'éducation ou de la formation ne peuvent compter que faiblement sur les institutions éducatives(écoles, commissions scolaires, ministère de l'Éducation) ou encore les entreprises et organismes de formation pour développer leur identité professionnelle, mais doivent plutôt construire celle-ci à partir de l'interprétation de leur expérience de travail. Ainsi, la volonté affichée par le gouvernement du Québec de professionnaliser les différents métiers de l'éducation et de la formation, et donc de réformer la formation qui est censée y conduire, devrait s'appuyer sur la mise en place de contextes formatifs qui pourraient accompagner et soutenir les futurs professionnels dans un processus de mise en récit de soi visant à interpréter leurs expériences de travail, c'est-à-dire leur permettant de donner sens aux différentes situations professionnalisantes rencontrées.

\section{BIBLIOGRAPHIE}

Bancel, D. (1989). Créer une nouvelle dynamique de la formation des maîtres. Rapport présenté au ministre d'État, ministre de l'Éducation nationale, de la Jeunesse et des Sports, France. Repéré à http://formerlesformateurs.free.fr/rapport_bancel_10oct2006.html Barbier, J.-M. (1996). Savoirs théoriques et savoirs d'action. Paris : Presses universitaires de France. Barreau, J.-M. (2001). Vichy contre l'école de la République. Paris : Flammarion. Baudouin, J.-M., \& Friedrich, J. (dir.) (2001). Théories de l'action et éducation. Bruxelles : De Boeck. Boulet, M. (2001). La formation des acteurs de l'agriculture : continuité et ruptures 1945-1985. Dijon : Éducagri-ENESAD. 
Bourdieu, P. (1989). La noblesse d'état. Paris : Les éditions de minuit.

Bourdoncle, F. (1991). La professionnalisation des enseignants : Analyses sociologiques anglaises et américaines. La fascination des professions. Revue française de pédagogie, 94, 73-91.

Bru, M. (2002). Pratiques enseignantes : des recherches à conforter et à développer. Revue française de pédagogie, 138, 63-73.

Carbonneau, M. (1993). La professionnalisation de l'enseignement et de la formation des enseignants.Revue des sciences de l'éducation, 19(1), 33-57.

Champy, F. (2009). La sociologie des professions. Paris : Presses universitaires de France.

Charlot, B. (1997). Du rapport au savoir : Éléments pour une théorie. Paris : Anthropos.

Chevallard, C. (1980). La transposition didactique : du savoir savant au savoir enseigné. Grenoble : La pensée sauvage.

Clot, Y. (2006). Clinique du travail et clinique de l'activité. Nouvelle revue de psychosociologie, 1 , 165-177.

Collectif IUFM d'Aix-Marseille (2009). La professionnalité de l'enseignant : la place des savoirs, quels savoirs, quelles compétences, et les conséquences pour la formation. Repéré à http:// www.etatsgeneraux-formationdesenseignants.fr/spip.php ?article262

Correa-Molina, E., \& Gervais, C. (2008). Les stages en formation à l'enseignement : pratiques et perspectives théoriques. Québec : Presses de l'Université du Québec.

Couturier, Y., \& Chouinard, I. (2008). La relation est-elle soluble dans la didactique ? La relationnalité dans les métiers relationnels comme objet d'une didactique des savoirs professionnels. Dans Y. Lenoir \& P. Pastré (dir.), Didactique professionnelle et didactiques disciplinaires en débat (p. 213-223). Toulouse : Octarès.

Fabre, M. (1994). Penser la formation. Paris : Presses universitaires de France.

Gouvernement du Québec (2001). La formation à l'enseignement : Les orientations, les compétences professionnelles. Québec : Ministère de l'Éducation.

Hasni, A., \& Lenoir, Y. (2006). Les disciplines, la didactique des disciplines et le curriculum de formation à l'enseignement primaire : de la maîtrise à l'adéquation. Dans Y. Lenoir \& M.-H. Bouillier-Oudot (dir.), Savoirs professionnels et curriculums de formation (p. 125-166). Québec : Les presses de l'Université Laval.

Illich, Y. (1977). Le chômage créateur. Paris : Le Seuil.

Joshua, S. (1996). Le concept de transposition didactique n'est-il propre qu'aux mathématiques? Dans C. Raisky \& M. Caillot (dir.), La didactique au delà des didactiques. Regards croisés sur des concepts fédérateurs (p. 61-73). Bruxelles : De Boeck.

Lang, V. (1999). La professionnalisation des enseignants. Paris : Presses universitaires de France.

Lenoir, Y., Laforest, M., \& Pellerin, B. (1995). Objets de formation privilégiés dans des curricula de formation des maîtres du primaire au Québec au cours des années quatre-vingt. Spirale, 16(3), 107-135.

Leplat, J. (1992). L'analyse du travail en psychologie ergonomique. Toulouse : Octarès.

Lesne, M. (1977). Travail pédagogique et formation d'adultes. Paris : Presses universitaires de France. 
Lessard, C., \& Tardif, M. (2005). La nature et la place d'une formation professionnelle selon les conceptions de l'université. DansY. Lenoir (dir.) Les professionnalités et les formations (p. 27-66). Bruxelles : De Boeck.

Malglaive, G. (1990). Enseigner à des adultes. Paris : Presses universitaires de France.

Martineau, S. (2007, juin). Analyse descriptive et compréhensive des pratiques d'enseignement : les routines sous l'œil de la phénoménologie inspirée de A. Schütz. Communication présentée dans le cadre de la demi-journée d'étude du CRIE et de la CRCIE, Sherbrooke, Québec, juin 2007.

Martineau, S. (2008). L'identité professionnelle et l'insertion professionnelle : essai de problématique. Formation et profession, 15(1), 40-43.

Mayen, P. (2008). Intégrer les savoirs à l'action. Dans P. Perrenoud, C. Lessard, M. Altet \& L. Paquay (dir.), Conflits de savoirs en formation des enseignants : entre savoirs issus de la recherche et savoirs issus de la recherche (p. 123-137). Bruxelles : De Boeck.

Mialaret, G. (1996). Savoirs théoriques, savoirs scientifiques et savoirs d'action en éducation. Dans J.-M. Barbier (dir.), Savoirs théoriques et savoirs d'action (p. 161-187). Paris : Presses universitaires de France.

OCDE. (2008). Regards sur l'éducation 2008. Paris : OCDE.

Pastré, P. (1997). Didactique professionnelle et développement. Psychologie Française, 42(1), 89-100. Perrenoud, P., Altet, M., Lessard, C \& Paquay, L. (dir.). (2008). Conflits de savoirs en formation des enseignants : entre savoirs issus de la recherche et savoirs issus de la recherche. Bruxelles : De Boeck.

Piot, T. (2009). Quels indicateurs pour mesurer le développement professionnel dans les métiers adressés à autrui ? Revue Questions vives en éducation et formation, 5(11), 259-275.

PRISME (2008). Professionnalité enseignante. Repéré à http://www.prisme-asso.org/

Raisky, C. (1993). Problème du sens des savoirs professionnels, préalable à une didactique. Dans $P$. Jonnaert \& Y. Lenoir (dir.), Sens des didactiques et didactique du sens (p. 101-121). Sherbrooke : Éditions du CRP.

Raisky, C. (1999). Complexité et didactique. Éducation permanente, 139, 37-63.

Raisky, C., \& Loncle, J.-C. (1993). Didactiser des savoirs professionnels : l'exemple des formations agronomiques. Dans P. Jonnaert \& Y. Lenoir (dir.), Sens des didactiques et didactique du sens (p. 339-366). Sherbrooke : Éditions du CRP.

Rogalski, J., \& Samurçay, R. (1994). Modélisation d'un savoir de référence et transposition didactique dans la formation de professionnels de haut niveau. Dans G. Arsac (dir.), La transposition didactique à l'épreuve (p. 35-71). Paris : La pensée sauvage.

Roger, L., \& Maubant, P. (2011). Les pratiques éducatives des enseignants : entre savoirs d'enseignement, savoirs d'expérience et savoirs professionnels. Dans P. Maubant (dir.), Enjeux de la place des savoirs dans les pratiques éducatives en contexte scolaire (p. 213-241). Québec: Presses de l'Université du Québec.

Schön, D. A. (1991) The Reflective Turn: Case Studies In and On Educational Practice. New York : Teachers Press, Columbia University.

Solar, C., \& Hébrard, P. (2008). Professionnalisation et formation des adultes. Paris : L'Harmattan.

Trottier, C., \& Lessard, C. (2002). La place de l'enseignement de la sociologie de l'éducation dans les programmes de formation des enseignants au Québec : étude de cas inspirée d'une sociologie du curriculum. Éducation et société, 9, 53-71. 
Vanhulle, S. (2008). Au cœur de la didactique professionnelle, la subjectivation des savoirs. Dans Y. Lenoir, \& P. Pastré (dir.), Didactique professionnelle et didactiques disciplinaires en débat. Toulouse : Octarès.

Vanhulle, S., \& Lenoir, Y. (2005). L'état de la recherche au Québec sur la formation à l'enseignement : Vers de nouvelles perspectives en recherche. Sherbrooke : Éditions du CRP.

Verret, M. (1975). Le temps des études. Paris : Librairie Honoré.

\section{RÉSUMÉS}

Les textes officiels et/ou professionnels sur les formations initiales et/ou continues dans les métiers adressés à autrui défendent parfois avec vigueur une visée: la professionnalisation. Outre que ce terme de professionnalisation pourrait apparaître comme un effet de mode, il semble important d'en comprendre ses fondements, ses conditions d'émergence tant du point de vue des recherches en sciences de l'éducation que du point de vue des discours sur et pour la professionnalisation, ses valeurs ajoutées, ses retombées attendues. Cet article propose donc au lecteur un tour d'horizon historique permettant d'expliquer le succès de cette notion. Il vise aussi à définir le terme en le confrontant à d'autres notions ou concepts, et notamment ceux de professionnalité et de savoirs professionnels. Enfin, il cherche à mettre en évidence les grandes questions et enjeux contenus dans cet objectif de professionnalisation en montrant aussi quelles peuvent être les conditions gagnantes pour faire d'un parcours de formation et de développement professionnel un véritable processus de professionnalisation des professionnels de l'éducation ou de la formation en devenir.

\section{INDEX}

Mots-clés : métiers de la formation, professionnalisation, professionnalité, savoirs professionnels

\section{AUTEURS}

\section{PHILIPPE MAUBANT}

Professeur titulaire

Directeur de l'Institut de recherche sur les pratiques éducatives (IRPÉ)

Philippe.Maubant@USherbrooke.ca

\section{LUCIE ROGER}

Doctorante en sciences de l'éducation

Université de Sherbrooke, local A10 3044

2500, boul. de l'Université

Sherbrooke (Québec) J1K 2R1

Lucie.Roger@USherbrooke.ca 